\title{
Traditional Chinese Medicine Tongxinluo Improves Cardiac Function of Rats with Dilated Cardiomyopathy
}

\author{
Fang-Fang Shen, ${ }^{1}$ Ting-Hui Jiang, ${ }^{2}$ Jin-Qi Jiang, ${ }^{1}$ Ying Lou, ${ }^{1}$ and Xu-Min $\mathrm{Hou}^{3}$ \\ ${ }^{1}$ Department of Emergency, Shanghai Chest Hospital, Shanghai Jiao Tong University, Shanghai 200030, China \\ ${ }^{2}$ Department of Integrated Traditional Chinese and Western Medicine, Shanghai Tong Ren Hospital, Shanghai 200050, China \\ ${ }^{3}$ Department of Cardiology, Shanghai Chest Hospital, Shanghai Jiao Tong University, Shanghai 200030, China \\ Correspondence should be addressed to Jin-Qi Jiang; jiangjqsci@163.com
}

Received 23 October 2014; Accepted 1 December 2014; Published 28 December 2014

Academic Editor: Kenji Watanabe

Copyright (C) 2014 Fang-Fang Shen et al. This is an open access article distributed under the Creative Commons Attribution License, which permits unrestricted use, distribution, and reproduction in any medium, provided the original work is properly cited.

\begin{abstract}
The study aimed at testing the hypothesis that tongxinluo capsule might exert its cardioprotective effect by preventing ventricular remodeling and improving coronary microvascular function in a rat model of doxorubicin-induced dilated cardiomyopathy (DCM). Rats that survived DCM induction were randomly divided into three groups to be given $1.5 \mathrm{~g} \cdot \mathrm{kg}^{-1} \cdot \mathrm{day}^{-1}$ (TXL-H, $n=9$ ) or $0.15 \mathrm{~g} \cdot \mathrm{kg}^{-1} \cdot \mathrm{day}^{-1}$ (TXL-L, $\left.n=10\right)$ of tongxinluo, or normal saline at the same volume (DCM-C, $\left.n=10\right)$ intragastrically. Age matched normal rats treated with normal saline were used as normal controls (NOR-C, $n=9$ ). After four weeks of treatment, the DCM-C, TXL-H, and TXL-L groups exhibited significant cardiac dysfunction, left ventricular remodeling, and coronary microvascular dysfunction, compared with the NOR-C rats. However, myocardial functional parameters were significantly improved and microvascular density (MVD) increased in the TXL-H group compared with the DCM-C group (all $P<0.01)$. Left ventricular remodeling was prevented. There were close linear relationships between CVF and LVEF $(r=-0.683$, $P<0.05)$, MVD and LVEF $(r=0.895, P<0.05)$, and MVD and CVF $(r=-0.798, P<0.05)$. It was indicated that high-dose tongxinluo effectively improved cardiac function in rat model of DCM.
\end{abstract}

\section{Introduction}

Dilated cardiomyopathy (DCM) is characterized by ventricular chamber enlargement and systolic dysfunction with normal ventricular wall thickness [1]. It is an important cause of sudden cardiac death and heart failure and is the leading indication for cardiac transplantation in children and adults worldwide [2]. Doxorubicin (Dox) is an effective antitumor agent. Despite its use as a common chemotherapeutic agent, Dox use can also lead to cardiotoxicity. Multiple intravenous Dox treatments over a period of several months have been shown to induce cardiomyopathy and chronic heart failure in humans [3]. Study has also shown that rats treated with $15 \mathrm{mg} / \mathrm{kg}$ Dox develop DCM [4].

Although cardiac remodeling characterizes the natural history of DCM [5-7] and some drugs have been reported to have inhibitory effects on left ventricular remodeling [8], the mechanisms involved in the progressive deterioration of cardiac function are largely unknown. As dysfunction of the coronary microvessels is present from very early onset of the disease $[9,10]$, it has been proposed that impairment of coronary microcirculation may be responsible for the marked decrease in coronary flow reserve (CFR) [11] and may cause progressive contractile impairment, ventricular dilation, and heart failure [12]. It has been demonstrated that the presence and extent of early stage coronary microvascular dysfunction in DCM is an independent and relevant predictor of worse prognosis [13]. On the basis of these studies it is becoming evident that the coronary microcirculation is involved in the pathogenesis of DCM and should be considered as a new target of treatment in DCM $[10,12,14]$.

Pharmaceutics of herbal medicine are undergoing rapid development in China. With the progression of modern technology, more and more herbal compound extracts are being authenticated, standardized, and administered successfully in clinical practice. Tongxinluo capsule is a compound preparation formulated on the meridian theory of traditional Chinese medicine and was officially approved for treatment of 
TABLE 1: Formulation of tongxinluo capsule.

\begin{tabular}{lcc}
\hline Components & $\begin{array}{c}\text { Voucher specimen } \\
\text { number }\end{array}$ & Part used \\
\hline Panax ginseng C.A. Mey. (extraction) & 11,001 & Root and rhizome \\
Paeonia lactiflora Pall. (extraction) & 11,003 & Root \\
Ziziphus jujuba Mill. Var. spinosa (Bunge) Hu ex H.F.Chou (extraction) & 11,002 & Seed \\
Santalum album L. (extraction) & 11,004 & 1.68 \\
Dalbergia odorifera T.C.Chen (extraction) & 11,005 & Heartwood of stem \\
Steleophaga plancyi (Boleny) (Microoryzae farina) & 12,003 & Heartwood of stem and root \\
Scolopendra subspinipes mutilans L. Koch (farina) & 12,001 & Female dried body \\
Hirudo nipponica Whitman (farina) & 12,004 & Dried body \\
Cryptotympana pustulata Fabricius (farina) & 12,005 & Dried body \\
Buthus martensii Karsch (farina) & 12,002 & Skin \\
Boswellia carteri (farina) & 11,006 & 27.62 \\
Borneolum syntheticum (artificial) & 11,007 & Dried body \\
\hline
\end{tabular}

angina pectoris and ischemic stroke in 1996 by the State Food and Drug Administration of China $[15,16]$. Pharmaceutical analysis has demonstrated that it contains multiple active components that may be responsible for multiple therapeutic effects, such as preventing ventricular remodeling $[17,18]$ and improving microvascular function $[19,20]$ in the brain and the heart. However, the result of these therapeutic effects on DCM has not been studied to date.

In the present study, we aimed at testing the hypothesis that tongxinluo might exert its cardioprotective effect by preventing ventricular remodeling and especially by improving coronary microvascular function in a rat model of doxorubicin-induced DCM.

\section{Materials and Methods}

2.1. Components and Fingerprint Chromatography of Tongxinluo Capsule. Tongxinluo capsule (authorized number: Z19980015, lot number: 101005) was provided by Shijiazhuang Yiling Pharmacy (Heibei, China). The herbal drugs were authenticated and standardized on marker compounds according to the Chinese Pharmacopoeia 2005 [21]. Tongxinluo contains 12 medicinal components (Table 1), which were ground to a superfine powder with diameters $\leq 10 \mu \mathrm{m}$ by a micronizer and prepared as capsules. To reduce dose variability of tongxinluo capsules among different batches, the species, origin, harvest time, medical parts, and concocted methods for each component were strictly standardized. Moreover, high performance liquid chromatography (HPLC) and gas chromatography (GC) for fingerprint analysis were applied to quantitate the components of the tongxinluo capsule $[15,22]$. The detailed methods and results are described in the Supplementary Material available online at http://dx.doi.org/10.1155/2014/323870.

2.2. Animal Models. Doxorubicin-induced DCM was generated as described previously [23]. Briefly, fifty-four male Sprague-Dawley rats weighing $220 \pm 10 \mathrm{~g}$ (Shanghai SLAC Laboratory Animal Co., Ltd.) were used in this study. After observation for one week in the normal laboratory environment on an ordinary diet, forty-five animals were administered doxorubicin hydrochloride (Zhejiang Haizheng Pharmaceutical Industry, China) intraperitoneally in six equal injections (each containing $2.5 \mathrm{mg} / \mathrm{kg}$ ) over a period of 2 weeks for a total cumulative dose of $15 \mathrm{mg} / \mathrm{kg}$. The remaining nine received the same volume of normal saline for the control. Two weeks after cessation of doxorubicin injection, both groups of rats were examined by transthoracic echocardiography to see whether the model was successful. All animals were treated humanely in accordance with the Principles of Laboratory Animal Care formulated by the Guide for the Care and Use of Laboratory Animal, published by the US National Institutes of Health. The protocol was approved by the Ethics Committee of Shanghai Chest Hospital Affiliated to Shanghai Jiao Tong University.

2.3. Grouping and Treatment. Sixteen (35.6\%) of the 45 doxorubicin-administrated rats died from day 14 to 27. Animals that survived after DCM induction were randomly divided into three groups: (1) the high-dose tongxinluo group (TXL-H, $n$ = 9): received tongxinluo superfine powder intragastrically at a dosage of $1.5 \mathrm{~g} \cdot \mathrm{kg}^{-1} \cdot \mathrm{day}^{-1}$ for 4 weeks; (2) the low-dose tongxinluo group (TXL-L, $n=10$ ): received tongxinluo superfine powder intragastrically at a dosage of $0.15 \mathrm{~g} \cdot \mathrm{kg}^{-1} \cdot \mathrm{day}^{-1}$ for 4 weeks $[24,25]$; (3) the DCM control group (DCM-C, $n=10$ ): received normal saline intragastrically at the same volume as the tongxinluo-treated groups for 4 weeks. Age-matched normal rats treated with normal saline intragastrically were used as normal controls (NOR-C, $n=9$ ). Throughout the treatment, the general appearance, behavior, body weight, and survival rate of the rats were observed every day.

2.4. Echocardiography Measurements. Before and after the treatment, transthoracic echocardiography was performed in all groups under chloral hydrate anesthesia in a supine position using a high-resolution small animal echocardiographic system (VisualSonics, Canada) equipped with a $7.5-\mathrm{MHz}$ 
transducer. A two-dimensional short-axis view of the left ventricle was obtained at the level of the papillary muscle and two-dimensional targeted M-mode tracings were recorded. The detection index was as follows: left ventricular ejection fraction (LVEF), left ventricular end-systolic diameter (LVEDD), left ventricular end-systolic diameter (LVESD), and left ventricular fractional shortening (LVFS). All of the parameters were measured over 3 consecutive cardiac cycles.

2.5. Tissue Sample Preparation. After echocardiography measurement, hearts were arrested in diastole by intravenous injection of $\mathrm{KCl}(2 \mathrm{~mol} / \mathrm{L})$. The wet myocardium was isolated and weighed to calculate the ratio of heart weight (HW) to body weight (BW). The excised myocardium was sliced into three blocks (basal, mid-region, and apical) and the mid-region portion was fixed in $4 \%$ paraformaldehyde for 16-18 hours at $4^{\circ} \mathrm{C}$ followed by embedding in paraffin wax and cutting into $5 \mu \mathrm{m}$ slices for subsequent histologic and immunohistochemical analysis.

\subsection{Histopathological Examination. Paraffin-embedded} slices were stained with hematoxylin-eosin (HE) for morphologic examination or picrosirius red for interstitial fibrosis determination (collagen staining). For $\mathrm{HE}$ slices, myocyte diameter measurements were performed in 10 myocytes selected per field in 200-fold magnification by light microscopy (Leica, Germany) [26]. Short axis diameters of each myocyte were measured from the hearts of all groups of rats. Each average value was obtained from the data for 10 myocytes and was used as independent sampling data. In addition, myocyte hypertrophy, inflammatory cell infiltrations, vacuolization, and the extent of vascular hyperemia were observed in $\mathrm{HE}$ sections. For collagen staining, An Axioplan II KS 400 microscope (Carl Zeiss, Germany) was used to capture at least 4 randomly selected images from each slide using the $\times 10$ objective. Collagen fibers were stained red or green under linearly polarized light, and the area of myocardial fibrosis in left ventricular tissue sections stained with picrosirius red was quantified using a color image analyzer (CIA-102, Olympus, Tokyo, Japan). The result was presented as interstitial collagen volume fraction (CVF) which was calculated as the sum of all connective tissue area divided by the sum of all connective tissue and cardiac myocyte areas, as demonstrated previously in a number of studies [27-29].

2.7. Immunohistochemistry. To detect the microvessels in the myocardium, endothelial cells were stained with the specific primary antibody to CD31 (1:50, ab28364, Abcam, Cambridge, UK), according to the product specification. Microvascular density (MVD) in the myocardium was determined by a double-headed light microscope (Leica, Germany) using the counting method introduced by Weidner [30]. Briefly, the myocardial area in rat specimens containing the maximum number of microvessels was identified by scanning at low power $(\times 40$ and $\times 100)$. Individual microvessels were then counted at $\times 400$ magnification, where one field is equivalent to $0.09 \mathrm{~mm}^{2}$. Each positive endothelial cell cluster of immunoreactivity in contact with the selected field was counted as an individual vessel in addition to the morphologically identifiable vessels with a lumen. The number of countable microvessels in five fields was recorded, and the average value was defined as MVD for the case. Assessment of MVD was done without knowledge of any pathological data and was performed by two technicians in our microscopy laboratory. Disagreements on what constituted a microvessel were resolved by consensus.

2.8. Statistical Analysis. Values are presented as mean \pm standard deviation (SD) for continuous variables and absolute number (percentage) for categorical variables. Numerical data were analyzed using One-Way analysis of variance (ANOVA) followed by least significant difference (LSD) as post-hoc, whereas categorical data were compared using a chi-square test. Kapla-Meier analysis was used to obtain survival curves. The Log-Rank test for pairwise over strata was used to compare the survival rates among groups, and the Pearson correlation coefficient, to assess the correlation between any two variables of CVF, MVD, and LVEF. Statistical analysis was performed using SPSS 13.0 statistical software (SPSS Inc., Chicago, Illinois, USA). A 2-tailed $P$ value of less than 0.05 was considered statistically significant.

\section{Results}

3.1. Tongxinluo Increased Survival Rates in Rats with DCM. The survival study started after the DCM model was successfully set up, and after completion of the tongxinluo treatment, cumulative survival rate was $88.9 \%$ (8/9), 90.0\% (9/10), 80.0\% $(8 / 10)$, and $100.0 \%(9 / 9)$ in the TXL-H, TXL-L, DCM-C, and NOR-C groups, respectively. Although rats in the tongxinluotreated groups showed a higher survival rate than that in the DCM-C group, no statistically significant differences were revealed among these three groups (all $P>0.05$, Figure 1). The rats died presumably from severe congestive heart failure, malignant arrhythmias, or refractory ascites, as reported previously in a number of studies [31].

3.2. High-Dose Tongxinluo Decreased Heart Weight and Ratio of Heart Weight to Body Weight in Rats with DCM. The body weight (BW), heart weight (HW), and ratio of heart weight to body weight $\left(\mathrm{HB}^{-1}\right)$ are shown in Figure 2 . The BW was significantly larger in the NOR-C group than that in the TXL-H, TXL-L, and DCM-C groups $(411.64 \pm 13.86 \mathrm{~g}$ versus $383.89 \pm 11.35 \mathrm{~g}, 377.50 \pm 14.72 \mathrm{~g}, 374.53 \pm 10.73 \mathrm{~g}$, respectively, all $P<0.01$ ), respectively, but it did not differ among the latter three groups (all $P>0.05$ ). The HW and $\mathrm{H} \mathrm{B}^{-1}$ were significantly larger in the DCM-C group (1.22 \pm $0.05 \mathrm{~g}$ and $\left.3.24 \pm 0.09 \mathrm{~g} \cdot \mathrm{kg}^{-1}\right)$ than those in the NOR-C group $\left(1.06 \pm 0.06 \mathrm{~g}\right.$ and $2.58 \pm 0.08 \mathrm{~g} \cdot \mathrm{kg}^{-1}$, both $\left.P<0.01\right)$. The $\mathrm{HW}$ and $\mathrm{HB}^{-1}$ were significantly decreased in the TXL-H group $\left(1.14 \pm 0.06 \mathrm{~g}\right.$ and $\left.2.97 \pm 0.13 \mathrm{~g} \cdot \mathrm{kg}^{-1}\right)$ compared to the DCM-C group $\left(1.22 \pm 0.05 \mathrm{~g}\right.$ and $3.24 \pm 0.09 \mathrm{~g} \cdot \mathrm{kg}^{-1}$, both $P<0.05)$. No statistically significant differences were shown in $\mathrm{HW}$ and $\mathrm{HB}^{-1}$ between the TXL-L group $(1.20 \pm 0.05 \mathrm{~g}$ and $\left.3.16 \pm 0.08 \mathrm{~g} \cdot \mathrm{kg}^{-1}\right)$ and the DCM-C group $(1.22 \pm 0.05 \mathrm{~g}$ and $3.24 \pm 0.09 \mathrm{~g} \cdot \mathrm{kg}^{-1}$, both $\left.P>0.05\right)$. 
TABLE 2: Echocardiography results in each group before and after treatment.

\begin{tabular}{llcccc}
\hline Groups & & LVEDD & LVESD & LVEF & LVFS \\
\hline \multirow{2}{*}{ TXL-H } & (b) & $6.71 \pm 0.27^{* *}$ & $3.77 \pm 0.31^{* *}$ & $65.93 \pm 5.17^{* *}$ & $39.62 \pm 4.34^{* *}$ \\
& (a) & $6.59 \pm 0.26^{* *}$ & $3.57 \pm 0.37^{* *, \# \#}$ & $70.00 \pm 3.98^{* *, \# \#}$ & $41.98 \pm 3.43^{* *, \# \#}$ \\
\hline \multirow{2}{*}{ TXL-L } & (b) & $6.66 \pm 0.25^{* *}$ & $3.80 \pm 0.33^{* *}$ & $66.69 \pm 3.04^{* *}$ & $39.94 \pm 1.80^{* *}$ \\
& (a) & $6.82 \pm 0.18^{* *}$ & $3.90 \pm 0.21^{* *}$ & $63.32 \pm 3.37^{* *}$ & $37.89 \pm 2.77^{* *}$ \\
\hline \multirow{2}{*}{ DCM-C } & (b) & $6.70 \pm 0.38^{* *}$ & $3.82 \pm 0.37^{* *}$ & $62.79 \pm 6.07^{* *}$ & $36.34 \pm 5.05^{* *}$ \\
& (a) & $6.85 \pm 0.40^{* *}$ & $4.06 \pm 0.34$ & $61.57 \pm 6.17$ & $35.16 \pm 5.06$ \\
\hline \multirow{2}{*}{ NOR-C } & (b) & $6.05 \pm 0.26$ & $2.00 \pm 0.30$ & $90.88 \pm 1.66$ & $64.90 \pm 4.00$ \\
& (a) & $6.15 \pm 0.24$ & $2.04 \pm 0.31$ & $91.40 \pm 2.35$ & $64.80 \pm 4.62$ \\
\hline
\end{tabular}

LVEDD, left ventricular end-diastolic diameter; LVESD, left ventricular end-systolic diameter; LVEF, left ventricular ejection fraction; LVFS, left ventricular fractional shortening. ${ }^{* *} P<0.01$ versus the NOR-C group. ${ }^{\# \#} P<0.01$ versus the DCM-C group. b: before treatment; a: after treatment.

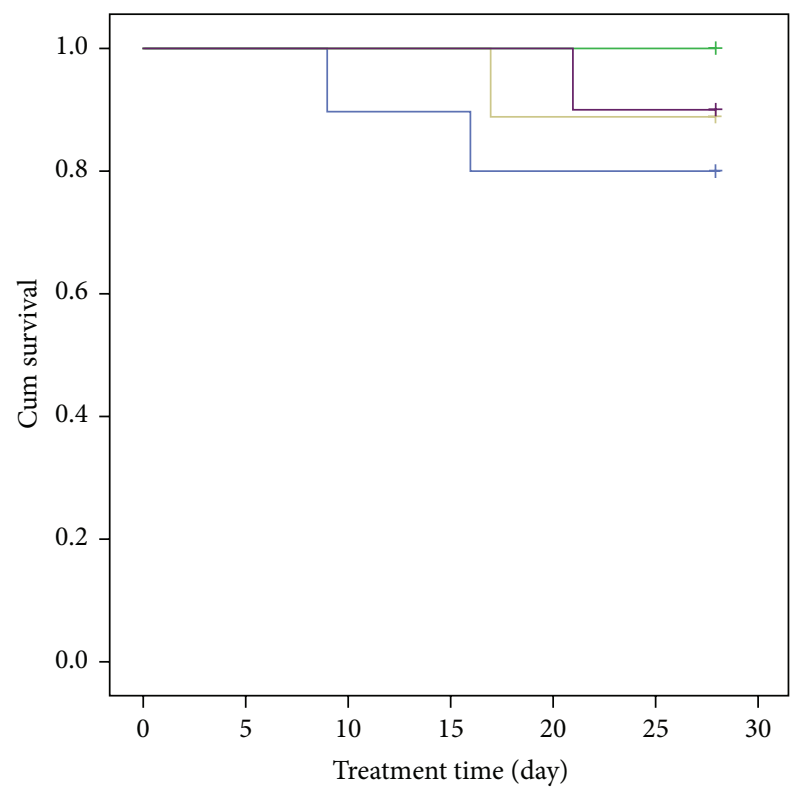

Group

$\begin{array}{ll}\neg \text { DCM-C } & + \text { DCM-C-censored } \\ \neg \text { NOR-C } & + \text { NOR-C-censored } \\ \neg \text { TXL-H } & + \text { TXL-H-censored } \\ \neg \text { TXL-L } & + \text { TXL-L-censored }\end{array}$

FIGURE 1: Effects of tongxinluo on cumulative survival rate. TXL$\mathrm{H}$ : high-dose tongxinluo group; TXL-L: low-dose tongxinluo group; DCM-C: DCM control group; NOR-C: normal control group.

3.3. High-Dose Tongxinluo Improved Cardiac Function in Rats with DCM. Figure 3 shows a representative echocardiography view of hearts from each group after treatment. As shown in Table 2, echocardiography analyses before treatment indicated that, compared with those in the NOR-C group, rats in the DCM-C, TXL-H, and TXL-L groups exhibited significant left ventricular dilation and systolic dysfunction, as evidenced by significantly increased LVEDD and LVESD (both $P<$ 0.01 ), and significantly decreased LVEF and LVFS (both $P<0.01)$. However, the LVESD, LVEF, and LVFS in the TXL-H group were significantly improved after treatment when compared with the DCM-C group (all $P<0.01$ ), while no significant difference in the four values was shown between the TXL-L group and DCM-C group (all $P>0.05$ ), demonstrating that high-dose tongxinluo treatment could markedly ameliorate cardiac dysfunction.

3.4. High-Dose Tongxinluo Improved Left Ventricle Remodeling in Rats with DCM. Cardiac tissue sections were examined using HE staining for morphological analysis. The myocyte size in the DCM-C group was significantly larger than that in the NOR-C group $(24.86 \pm 3.69$ versus $14.15 \pm 2.04$, $P<0.01)$. However, the TXL-H group had significantly reduced myocyte size when compared with the DCM-C group $(19.89 \pm 2.20$ versus $24.86 \pm 3.69, P<0.01)$, while there was no significant difference between the TXL-L group and DCM-C group $(22.75 \pm 3.00$ versus $24.86 \pm 3.69, P>0.05)$ (Figure $4(\mathrm{a}))$. The representative HE staining images $(\times 200)$ were shown in Figure 4(b). Obvious cardiac hypertrophy, vacuolar degeneration and local vascular hyperemia were detected in the hearts of the DCM-C group. However, the extent of pathological changes in the myocardial samples from the TXL-H group was less severe than that in the DCMC group.

Figure 5 illustrates the quantification of picrosirius red staining (Figure 5(a)) and shows representative images of left ventricular interstitial fibrosis using picrosirius red staining from each group (Figure 5(b)). Specifically, quantitative analysis revealed a significantly higher extent of left ventricular interstitial fibrosis in the DCM-C group when compared with the NOR-C group $(19.47 \pm 3.56$ versus $7.57 \pm 1.24$, $P<0.01)$. However, compared with the DCM-C group, high-dose tongxinluo administration significantly alleviated fibrotic deposits in the left ventricular wall $(10.18 \pm 2.09$ versus $19.47 \pm 3.56, P<0.01$ ), while the TXL-L group showed a tendency to lower CVF $(17.40 \pm 2.01$ versus $19.47 \pm 3.56$, $P>0.05)$.

3.5. High-Dose Tongxinluo Increased Microvascular Density in Rats with DCM. The values for microvessel counts and the representative images in each group after treatment are given in Figure 6. Overall, when compared with that of the NOR-C group, the MVD in myocardium was significantly decreased in the TXL-H, TXL-L, and DCM-C groups $(101.44 \pm 7.81$ versus 55.38 $\pm 6.74,42.44 \pm 5.03,36.00 \pm 7.15$, respectively; all $P<0.01)$, respectively. However, the MVD was significantly 


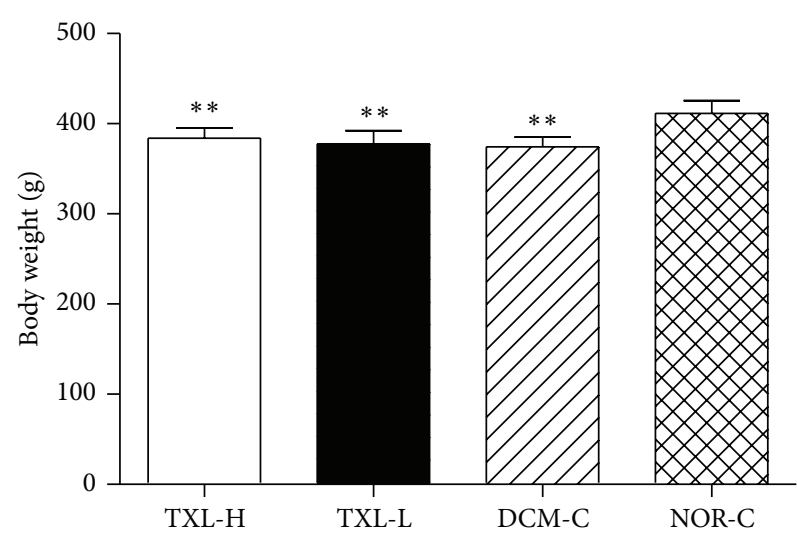

(a)

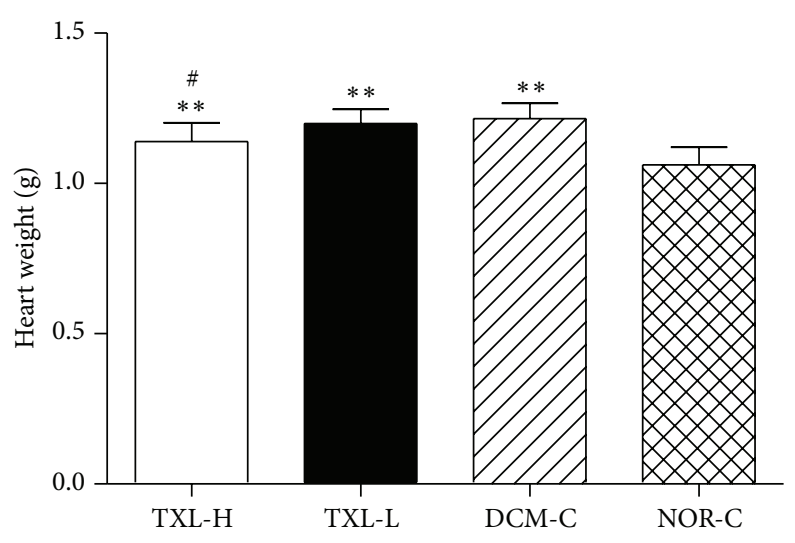

(b)

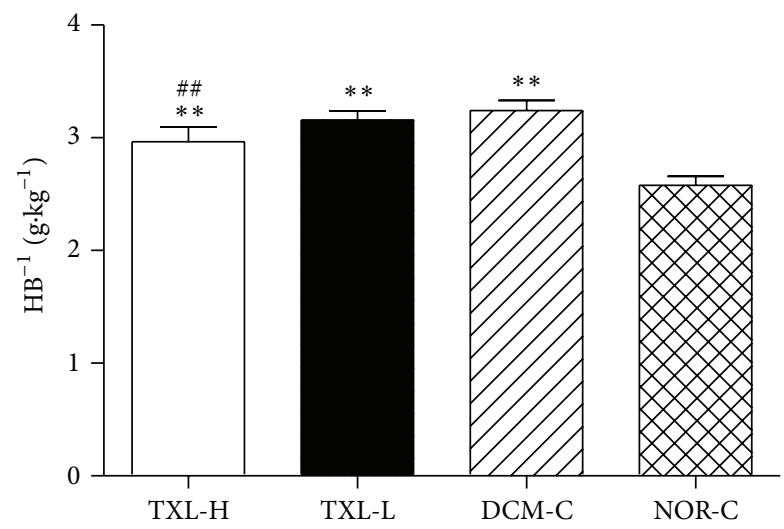

(c)

FiguRE 2: Effects of tongxinluo on body weight (a), heart weight (b), and ratio of heart weight to body weight $\left(\mathrm{H} \mathrm{B}^{-1}\right)(\mathrm{c})$. Values are mean \pm SD. ${ }^{* *} P<0.01$ versus the NOR-C group. ${ }^{\#} P<0.05$ and ${ }^{\# \#} P<0.01$ versus the DCM-C group.

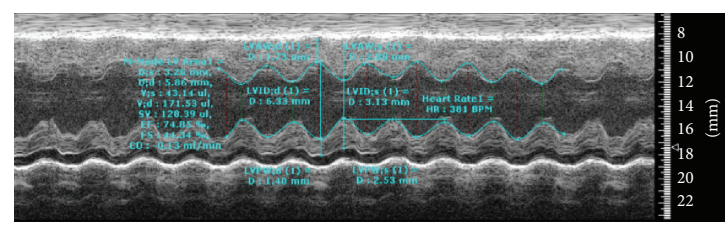

(a)

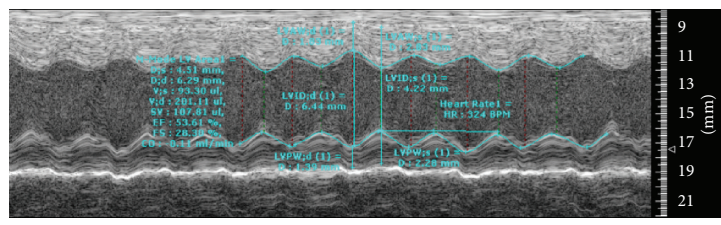

(c)

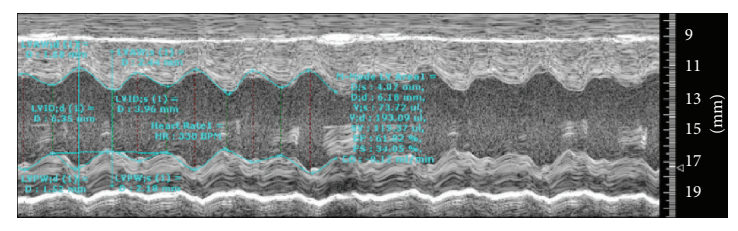

(b)

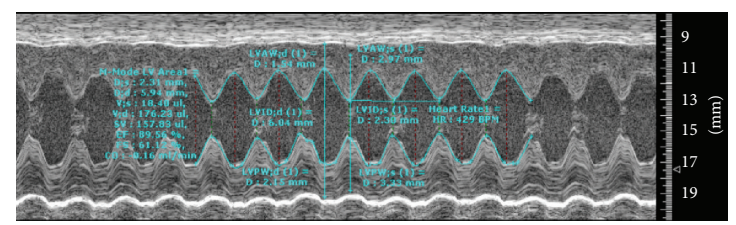

(d)

FIGURE 3: Representative echocardiographic views of a left ventricle from each group. (a) the TXL-H group; (b) the TXL-L group; (c) the DCM-C group; (d) the NOR-C group.

increased in the TXL-H group compared to the DCM-C group $(55.38 \pm 6.74$ versus $36.00 \pm 7.15, P<0.01)$ while no significant difference was found between the TXL-L group and DCM-C group $(42.44 \pm 5.03$ versus $36.00 \pm 7.15, P>$ $0.05)$, which demonstrated the dose-dependent angiogenesispromoting effect of tongxinluo.
3.6. The Correlations between CVF and LVEF, and CVF and LVEF Were Negative While MVD and LVEF Were Positively Correlated. Correlations between any two variables of CVF, MVD, and LVEF are shown in Figure 7. Overall, there were significant negative correlations between CVF and LVEF ( $r=$ $-0.683, P<0.05)$, and MVD and CVF $(r=-0.798$, 


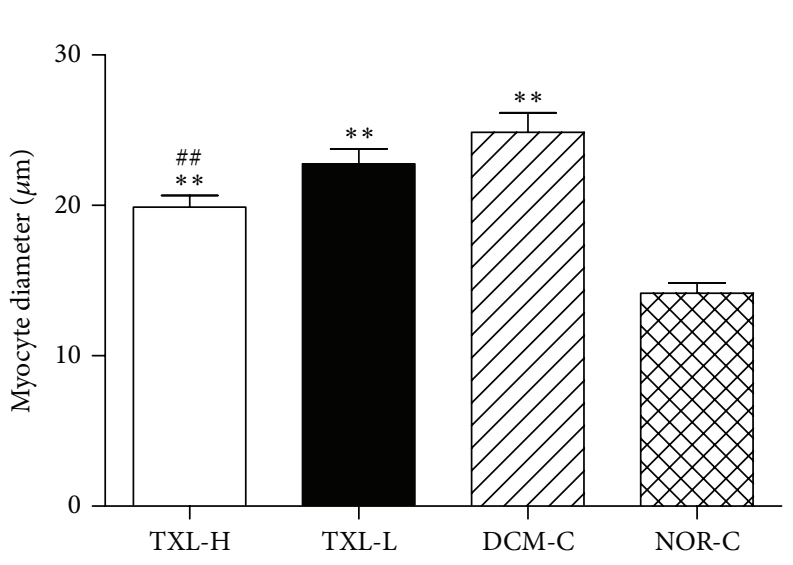

(a)

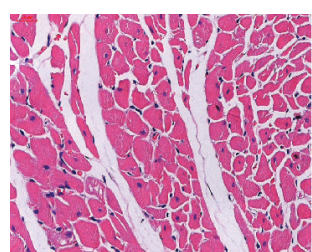

(A)

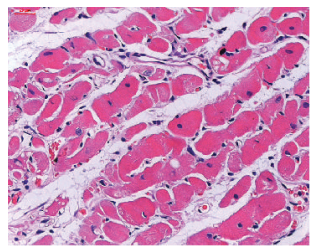

(C)

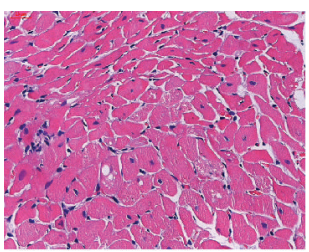

(B)

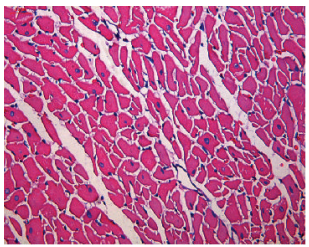

(D)

(b)

FIGURE 4: The effect of tongxinluo on myocardial morphology. (a) Quantitative analysis of myocyte diameter of the cross-sectional tissue slices of hearts. Values are mean $\pm \mathrm{SD} .{ }^{* *} P<0.01$ versus the NOR-C group. ${ }^{\# \#} P<0.01$ versus the DCM-C group. (b) Representative HE staining images $(\times 200)$. ((b)-(A)) the TXL-H group; a little of vacuolar degeneration and myocyte hypertrophy. ((b)-(B)) the TXL-L group; myocyte hypertrophy, vacuolar degeneration, and inflammation cells infiltration. ((b)-(C)) The DCM-C group; obvious myocyte hypertrophy, numerous vacuolar degeneration and local vascular hyperemia. ((b)-(D)) the NOR-C group; the normal heart.

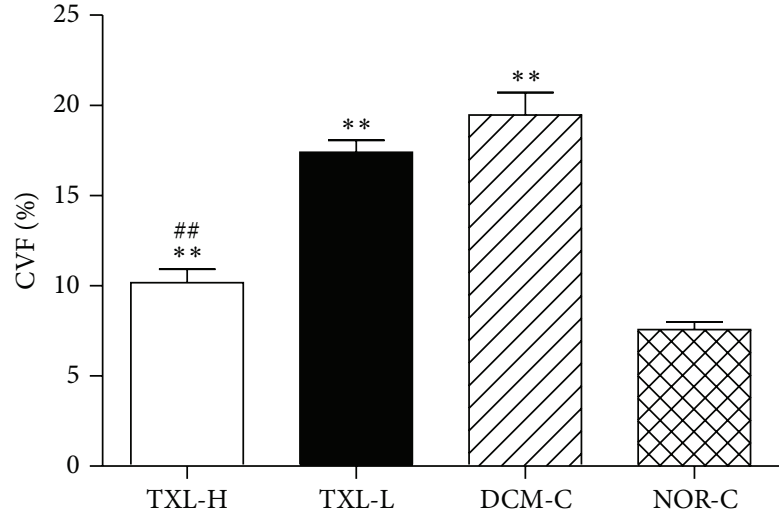

(a)

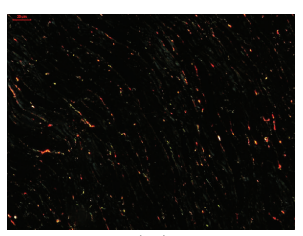

(A)

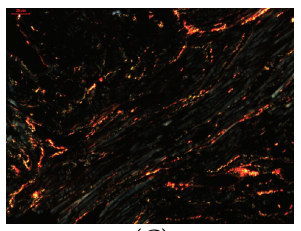

(C)

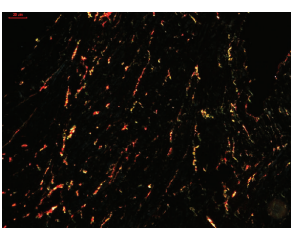

(B)

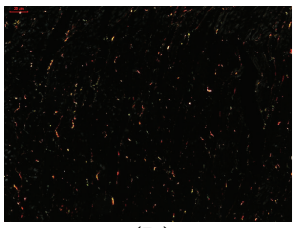

(D)

(b)

FIGURE 5: The effect of tongxinluo on left ventricular interstitial fibrosis. (a) Quantification of picrosirius red staining after treatment. Values are mean \pm SD. CVF, collagen volume fraction. ${ }^{* *} P<0.01$ versus the NOR-C group. ${ }^{\# \#} P<0.01$ versus the DCM-C group. (b) Representative images using picrosirius red staining from each group at $\times 10$ objective magnification. ((b)-(A)) the TXL-H group; ((b)-(B)) the TXL-L group; ((b)-(C)) the DCM-C group; ((b)-(D)) the NOR-C group.

$P<0.05)$, respectively. A significant positive correlation was also observed between MVD and $\operatorname{LVEF}(r=0.895, P<0.05)$.

\section{Discussion}

In the present study, using a rat model of dilated cardiomyopathy induced by doxorubicin hydrochloride in our laboratory, we examined the beneficial effects of tongxinluo on progression of cardiac dysfunction with dilated cardiomyopathy. We found that high-dose tongxinluo $\left(1.5 \mathrm{~g} \cdot \mathrm{kg}^{-1} \cdot \mathrm{day}^{-1}\right)$ attenuated left ventricular remodeling and promoted angiogenesis more effectively than low-dose tongxinluo $\left(0.15 \mathrm{~g} \cdot \mathrm{kg}^{-1} \cdot \mathrm{day}^{-1}\right)$.
The tongxinluo capsule is an officially approved treatment in China for angina pectoris and ischemic stroke and the compound preparation was formulated on the basis of the meridian theory of traditional Chinese medicine $[15,16]$. The routine clinical dose range of tongxinluo capsule (3 capsules, 3 times per day) approximates to $60 \mathrm{mg} \cdot \mathrm{kg}^{-1} \cdot \mathrm{day}^{-1}$ of tongxinluo powder, which may correspond to the dose of $400 \mathrm{mg} \cdot \mathrm{kg}^{-1} \cdot \mathrm{day}^{-1}$ in rats by taking account of the ratio of 6.3 [25]. In published experimental studies in rats, the highest dose effective for cardiac dysfunction was $1.5 \mathrm{~g} \cdot \mathrm{kg}^{-1} \cdot$ day $^{-1}$ [24]. Thus, the higher dose of $1.5 \mathrm{~g} \cdot \mathrm{kg}^{-1} \cdot \mathrm{day}^{-1}$ used in our study corresponds to $225 \mathrm{mg} \cdot \mathrm{kg}^{-1} \cdot \mathrm{day}^{-1}$ of tongxinluo powder, a dose approximately four times as high as the routine dose of $60 \mathrm{mg} \cdot \mathrm{kg}^{-1} \cdot \mathrm{day}^{-1}$ for patients. 


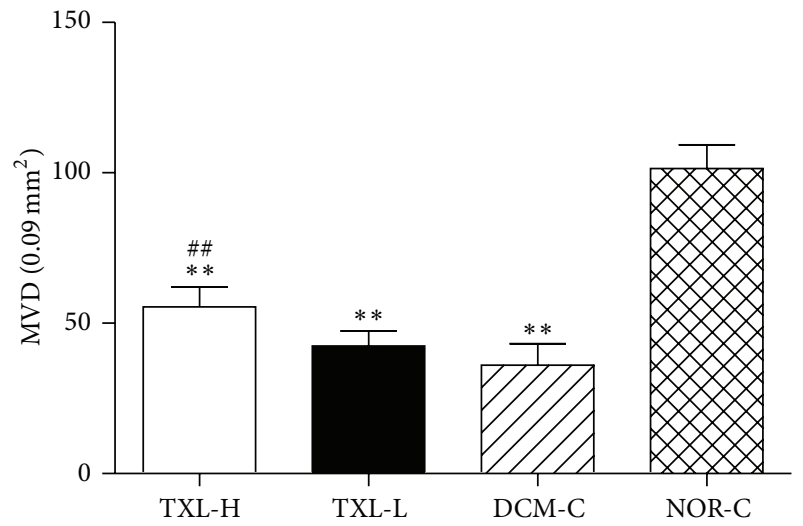

(a)

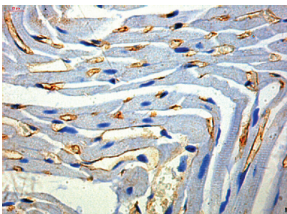

(A)

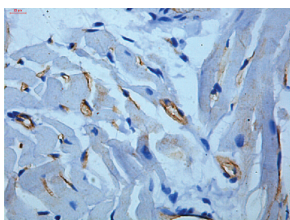

(C)

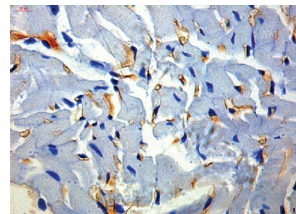

(B)

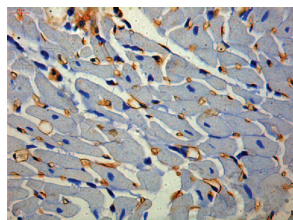

(D)

(b)

Figure 6: The effect of tongxinluo on angiogenesis. (a) Quantitative analysis of microvessel counts after treatment. Values are mean \pm SD. MVD, microvascular density. ${ }^{* *} P<0.01$ versus the NOR-C group. ${ }^{\#} P<0.01$ versus the DCM-C group. (b) Representative CD31 staining images $(\times 400)$. ((b)-(A)) the TXL-H group; ((b)-(B)) the TXL-L group; ((b)-(C)) the DCM-C group; ((b)-(D)) the NOR-C group.

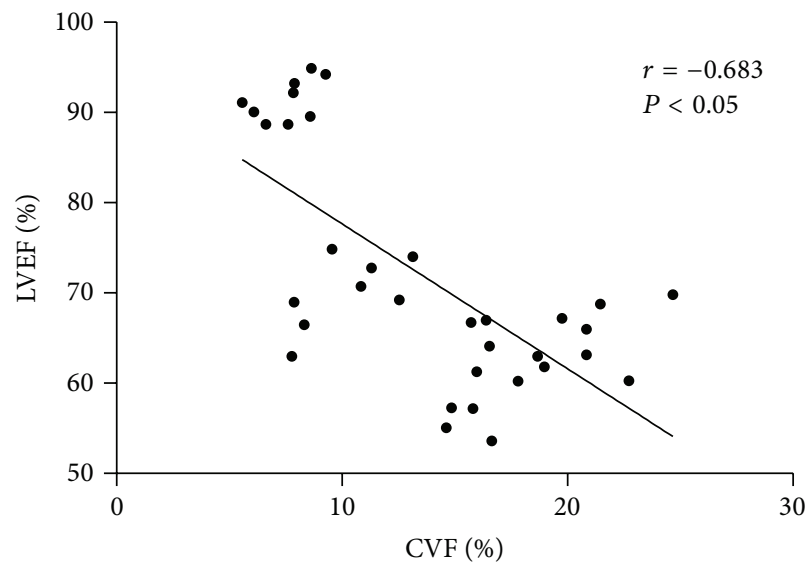

(a)

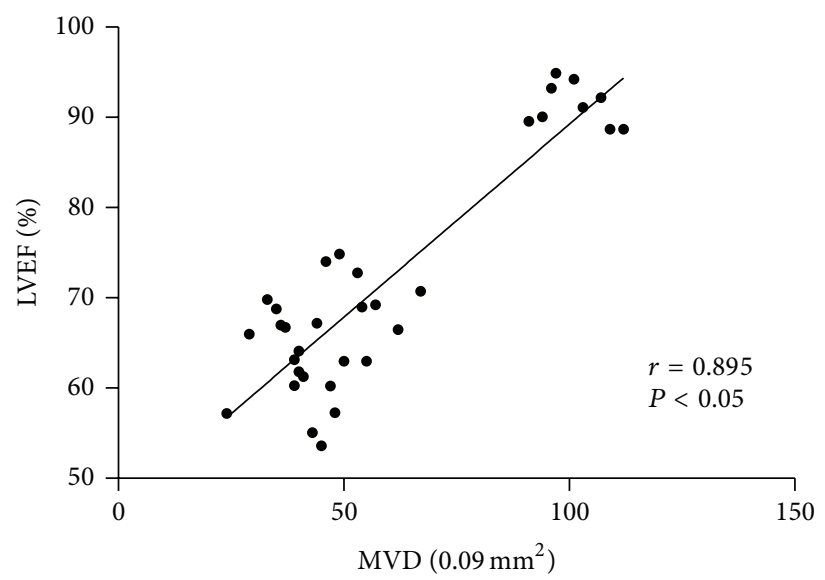

(b)

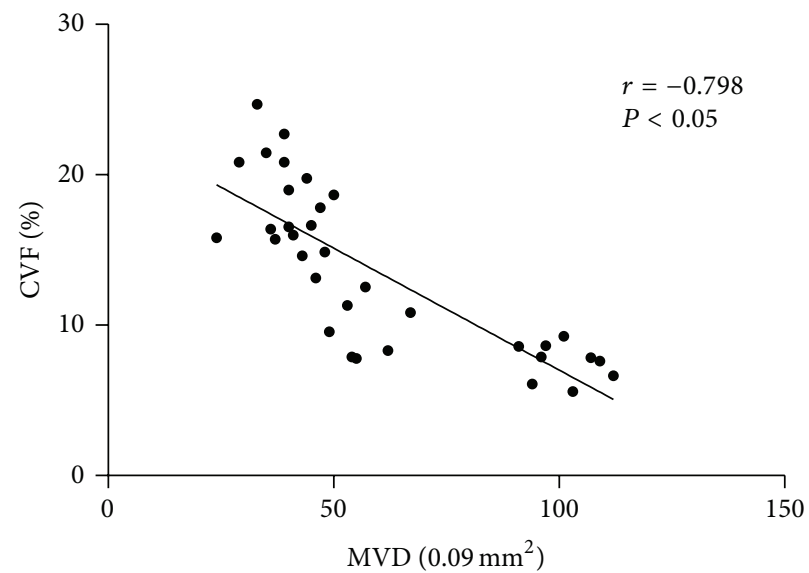

(c)

FIGURE 7: Relationships between collagen volume fraction (CVF), microvascular density (MVD), and left ventricular ejection fraction (LVEF). (a) Correlation between CVF and LVEF; (b) correlation between MVD and LVEF; (c) correlation between MVD and CVF. CVF, collagen volume fraction; MVD, microvascular density; LVEF, left ventricular ejection fraction. 
The lower dose of $0.15 \mathrm{~g} \cdot \mathrm{kg}^{-1} \cdot \mathrm{day}^{-1}$ in our study corresponds to $22.5 \mathrm{mg} \cdot \mathrm{kg}^{-1} \cdot \mathrm{day}^{-1}$ of tongxinluo powder, similar to one third of the routine clinical dose.

To our knowledge, ventricular remodeling is characterized by both quantitative and qualitative alterations of cardiac extracellular matrix and hypertrophy of cardiomyocytes [17]. Some basic studies have demonstrated that tongxinluo could inhibit ventricular remodeling in animals with spontaneous hypertension or myocardial infarction [17, 18, 32]. In our study, using two different doses, we found that tongxinluo capsule dose-dependently reduced the myocyte size in rats with DCM. In addition, the collagen volume fraction, an important index of cardiac fibrosis, was significantly lower in the TXL-H group than the DCM-C group, and histopathologic examinations showed that the degree of fibrosis was obviously lower when treated with high-dose tongxinluo. These effects of tongxinluo were associated with the prevention of ventricular remodeling. In a review of the literature, the authors have reported that ventricular remodeling could importantly affect the function of the ventricle [33], and Montera et al. [34] demonstrated a significant negative correlation between CVF and LVEF with correlation coefficient of 0.7 , which is a little more than that in our study $(r=-0.683$, $P<0.05)$. Therefore, the ventricular remodeling-preventing effects of tongxinluo may contribute to the improvement of cardiac function in rats with DCM.

More recent studies have shown that the coronary microcirculation may be directly affected in cardiomyopathies. Abnormalities in coronary flow reserve are often observed in patients with DCM [35-38]. Similar findings commonly suggest the presence of coronary artery stenosis, which will not be confirmed at cardiac catheterization in these patients, but abnormalities at the coronary microvascular level might similarly be responsible for these observations. Structural alternations of the coronary microcirculation, known as coronary remodeling, including incomplete arteriolar wall [39], as well as decreased microvessel density $[14,39,40]$, provide an anatomical basis for a decreased coronary flow reserve in DCM. Furthermore, Tsagalou et al. [11] observed a close linear relationship between CFR and MVD in patients with idiopathic dilated cardiomyopathy ( $r=0.756, P=0.0001)$, suggesting the important role of MVD in the decreased CFR and even the progression of cardiac dysfunction. Similarly, the impairment of MVD in the myocardium was also observed in our study. However, high-dose tongxinluo administration significantly increased MVD in the myocardium, demonstrating the angiogenesispromoting effect of tongxinluo. As evidenced by the strong correlation between MVD and LVEF $(r=0.895, P<0.05)$, this tongxinluo effect may also contribute to the improvement of cardiac function in rats with DCM.

Furthermore, a number of studies have suggested that changes of microvascular dysfunction play an important role in the development of ventricular remodeling. Particularly, the possibility was raised that microvascular dysfunction in the myocardium may be a powerful and independent predictor contributing to the remodeling of left ventricle [41-43]. This has been emphasized in a study demonstrating that the improvement of microvascular function early after myocardial infarction is beneficial in preventing left ventricular remodeling [42]. Also supporting our findings of microvascular dysfunction, MVD and CVF have a strong relationship $(r=-0.798, P<0.05)$, and left ventricular remodeling attenuates beneficially after promoting angiogenesis by treatment with high-dose tongxinluo.

However, the specific mechanisms of the angiogenesispromoting effects of tongxinluo in our study are not clear but may be implied from previous studies. In a model of focal cerebral ischemia, Chang et al. [19] demonstrated that tongxinluo could significantly improve the MVD of ischemic stroke rats, which may be related to the increase of NO and VEGF expression. Hu et al. [44] found that tongxinluo could induce angiogenesis in bone marrow mesenchymal stem cells (MSCs), and the underlying mechanisms are associated with increased migration ability of MSCs and the upregulation of MMP-2 and VEGF expressions. All of the above mechanisms of tongxinluo may contribute to the angiogenesis of the left ventricle of the rats with DCM in this investigation, although the exact mechanism needs further studies.

There are several limitations to this study. First, four weeks' administration of tongxinluo is a little short and that may be why a significant difference in LVEDD between the TXL-H group and DCM-C group was not observed, though the TXL-H group showed an obvious tendency to lower LVEDD. Second, the components of tongxinluo capsule were clear and pharmaceutical analysis has demonstrated that peoniflorin, ginsenoside Rg1, ginsenoside Rb1, jujuboside $\mathrm{A}$, and jujuboside $\mathrm{B}$ are the five most important active components in the tongxinluo capsule. However, it is not known which component is the most efficacious one responsible for the observed protective effect on cardiac dysfunction of the rats with DCM. We need to further fractionate the individual components of the compound and evaluate the protective effect of each individual component and their interactions. Third, although the beneficial effect of tongxinluo on cardiac function has been confirmed in our study, the detailed molecular mechanisms by which tongxinluo prevents ventricular remodeling and promotes angiogenesis require further investigation. Finally, human dilated cardiomyopathy is thought to have a variety of causes. Therefore, the clinical courses and pathological findings in this cardiomyopathy are not uniform. Thus, the present results only provide some small insight into the effectiveness of tongxinluo treatment for dilated cardiomyopathy.

\section{Conclusion}

In conclusion, the present study indicates that administration of high-dose tongxinluo effectively improves cardiac function in a rat model of doxorubicin-induced DCM, which may be attributed to prevention of left ventricular remodeling and, more particularly, promotion of angiogenesis by tongxinluo.

\section{Disclosure}

Fang-Fang Shen and Ting-Hui Jiang are co-first authors. 


\section{Conflict of Interests}

The authors declare that there is no conflict of interests regarding the publication of this paper.

\section{Acknowledgments}

The authors are greatly indebted to the participants for their participation in the study. This study was supported by a Grant from the Natural Science Fund of Shanghai, China (08ZR1417700).

\section{References}

[1] B. J. Maron, J. A. Towbin, G. Thiene et al., "Contemporary definitions and classification of the cardiomyopathies: an American Heart Association Scientific Statement from the Council on Clinical Cardiology, Heart Failure and Transplantation Committee; Quality of Care and Outcomes Research and Functional Genomics and Translational Biology Interdisciplinary Working Groups; and Council on Epidemiology and Prevention," Circulation, vol. 113, no. 14, pp. 1807-1816, 2006.

[2] N. K. Lakdawala, J. R. Winterfield, and B. H. Funke, "Dilated cardiomyopathy," Circulation: Arrhythmia and Electrophysiology, vol. 6, no. 1, pp. 228-237, 2013.

[3] G. Takemura and H. Fujiwara, "Doxorubicin-induced cardiomyopathy from the cardiotoxic mechanisms to management," Progress in Cardiovascular Diseases, vol. 49, no. 5, pp. 330-352, 2007.

[4] Q. Yu, Q. Li, R. Na et al., "Impact of repeated intravenous bone marrow mesenchymal stem cells infusion on myocardial collagen network remodeling in a rat model of doxorubicin-induced dilated cardiomyopathy," Molecular and Cellular Biochemistry, vol. 387, no. 1-2, pp. 279-285, 2014.

[5] A. Aleksova, G. Sabbadini, M. Merlo et al., "Natural history of dilated cardiomyopathy: from asymptomatic left ventricular dysfunction to heart failure-a subgroup analysis from the Trieste Cardiomyopathy Registry," Journal of Cardiovascular Medicine, vol. 10, no. 9, pp. 699-705, 2009.

[6] A. Di Lenarda, G. Secoli, A. Perkan et al., "Changing mortality in dilated cardiomyopathy," British Heart Journal, vol. 72, supplement 6, pp. S46-S51, 1994.

[7] G. M. Felker, R. E. Thompson, J. M. Hare et al., "Underlying causes and long-term survival in patients with initially unexplained cardiomyopathy," The New England Journal of Medicine, vol. 342, no. 15, pp. 1077-1084, 2000.

[8] M. Hamada, Y. Hara, T. Ohtsuka et al., "Effect of the addition of a $\beta$-blocker on left ventricular remodeling and prognosis in patients with dilated cardiomyopathy treated with angiotensinconverting enzyme inhibitor," Journal of Cardiovascular Pharmacology, vol. 41, no. 1, pp. S5-S10, 2003.

[9] J.-W. Chen, C.-T. Ting, Y.-H. Chen et al., "Differential coronary microvascular function in patients with left ventricular dysfunction of unknown cause-Implication for possible mechanism of myocardial ischemia in early stage of cardiomyopathy," International Journal of Cardiology, vol. 69, no. 3, pp. 251-261, 1999.

[10] D. Neglia, "New pathogenetic mechanisms in idiopathic dilated cardiomyopathy: pathophysiologic and prognostic role of coronary microvascular dysfunction," Recenti Progressi in Medicina, vol. 94, no. 6, pp. 247-252, 2003.
[11] E. P. Tsagalou, M. Anastasiou-Nana, E. Agapitos et al., "Depressed coronary flow reserve is associated with decreased myocardial capillary density in patients with heart failure due to idiopathic dilated cardiomyopathy," Journal of the American College of Cardiology, vol. 52, no. 17, pp. 1391-1398, 2008.

[12] D. Neglia and A. LAbbate, "Coronary microvascular dysfunction and idiopathic dilated cardiomyopathy," Pharmacological Reports, vol. 57, supplement, pp. 151-155, 2005.

[13] D. F. Neglia, M. Gallopin, and A. Giorgetti, "Prognostic role of coronary microvascular dysfunction in mild dilated cardiomyopathy," Circulation, vol. 96, supplement 8, p. I68, 1997.

[14] S. Roura, F. Planas, C. Prat-Vidal et al., "Idiopathic dilated cardiomyopathy exhibits defective vascularization and vessel formation," European Journal of Heart Failure, vol. 9, no. 10, pp. 995-1002, 2007.

[15] W. Q. Chen, L. Zhong, L. Zhang et al., "Chinese medicine tongxinluo significantly lowers serum lipid levels and stabilizes vulnerable plaques in a rabbit model," Journal of Ethnopharmacology, vol. 124, no. 1, pp. 103-110, 2009.

[16] L. Zhang, Y. Liu, X. T. Lu et al., “Traditional Chinese medication Tongxinluo dose-dependently enhances stability of vulnerable plaques: a comparison with a high-dose simvastatin therapy," The American Journal of Physiology-Heart and Circulatory Physiology, vol. 297, no. 6, pp. H2004-H2014, 2009.

[17] P.-L. Bu, X.-Q. Zhao, L.-L. Wang, Y.-X. Zhao, C.-B. Li, and Y. Zhang, "Tong-xin-luo capsule inhibits left venticular remodeling in spontaneously hypertensive rats by enhancing PPAR$\gamma$ expression and suppressing NF- $\kappa \mathrm{B}$ activity," Chinese Medical Journal, vol. 121, no. 2, pp. 147-154, 2008.

[18] W. Chen, X. Sun, W.-J. Wang et al., "Effects of tongxinluo capsule on cardiac ventricle remodeling after myocardial infarction: a multicentre clinical research," National Medical Journal of China, vol. 88, no. 32, pp. 2271-2273, 2008.

[19] L. P. Chang, Q. Y. Zhang, J. K. Han, C. Wei, and Z. H. Jia, "Experimental study on the interventional effects of Tongxinluo supermicropowder on angiogenesis of ischemic stroke rats," Chinese Pharmacological Bulletin, vol. 28, no. 7, pp. 1015-1018, 2012.

[20] S.-J. You, Y.-J. Yang, K.-J. Chen et al., “The protective effects of Tong-xin-luo on myocardium and microvasculature after reperfusion in acute myocardial infarction," Zhonghua Xin Xue Guan Bing Za Zhi, vol. 33, no. 5, pp. 433-437, 2005.

[21] National Pharmacopoela Committee, Pharmacopoeia of China, Chinese Publishing Company of Chimical Industry, Beijing, China, 2005.

[22] W. Su, A. Sun, D. Xu et al., “Tongxinluo inhibits oxidized lowdensity lipoprotein-induced maturation of human dendritic cells via activating peroxisome proliferator-activated receptor gamma pathway," Journal of Cardiovascular Pharmacology, vol. 56, no. 2, pp. 177-183, 2010.

[23] N. Siveski-Iliskovic, M. Hill, D. A. Chow, and P. K. Singal, "Probucol protects against adriamycin cardiomyopathy without interfering with its antitumor effect," Circulation, vol. 91, no. 1, pp. 10-15, 1995.

[24] H. J. Wang, L. D. Xie, E. H. Yao, and C. S. Xu, "Effect of tongxinluo on myocardial fibrosis in spontaneously hypertensive rats," Chinese Journal of Integrative Medicine on Cardio/Cerebrovascular Disease, vol. 1, no. 4, pp. 189-190, 2003.

[25] L. N. Xie, D. Y. Zeng, H. S. Zhang, D. M. Sun, X. F. Pang, and Q. G. Guan, "Effect of tongxinluo on vasoconstriction induced by the chronic injury of the adventitia in the rat carotid artery," Journal of Ethnopharmacology, vol. 131, no. 2, pp. 300-305, 2010. 
[26] V. Sukumaran, K. Watanabe, P. T. Veeraveedu et al., "Beneficial effects of olmesartan, an angiotensin II receptor type 1 antagonist, in rats with dilated cardiomyopathy," Experimental Biology and Medicine, vol. 235, no. 11, pp. 1338-1346, 2010.

[27] C. G. Brilla, J. S. Janicki, and K. T. Weber, "Impaired diastolic function and coronary reserve in genetic hypertension: role of interstitial fibrosis and medial thickening of intramyocardial coronary arteries," Circulation Research, vol. 69, no. 1, pp. 107115, 1991.

[28] C. G. Brilla, J. S. Janicki, and K. T. Weber, "Cardioreparative effects of lisinopril in rats with genetic hypertension and left ventricular hypertrophy," Circulation, vol. 83, no. 5, pp. 17711779, 1991.

[29] C. W. Doering, J. E. Jalil, J. S. Janicki et al., "Collagen network remodelling and diastolic stiffness of the rat left ventricle with pressure overload hypertrophy," Cardiovascular Research, vol. 22, no. 10 , pp. 686-695, 1988 .

[30] N. Weidner, "Current pathologic methods for measuring intratumoral microvessel density within breast carcinoma and other solid tumors," Breast Cancer Research and Treatment, vol. 36, no. 2, pp. 169-180, 1995.

[31] E. R. Schwarz, C. Pollick, J. Dow, M. Patterson, Y. Birnbaum, and R. A. Kloner, "A small animal model of non-ischemic cardiomyopathy and its evaluation by transthoracic echocardiography," Cardiovascular Research, vol. 39, no. 1, pp. 216-223, 1998.

[32] H.-Y. Qian, Y.-J. Yang, J. Huang et al., "Effects of Tongxinluofacilitated cellular cardiomyoplasty with autologous bone marrow-mesenchymal stem cells on postinfarct swine hearts," Chinese Medical Journal, vol. 120, no. 16, pp. 1416-1425, 2007.

[33] M. A. Pfeffer and E. Braunwald, "Ventricular remodeling after myocardial infarction: experimental observations and clinical implications," Circulation, vol. 81, no. 4, pp. 1161-1172, 1990.

[34] M. W. Montera, C. Drumond, C. Takiya, C. T. Mesquita, H. F. R. Dohmann, and C. Mady, "Correlation of myocardial interstitial collagen in the right ventricular septum with ventricular function of patients with ischemic cardiomyopathy," Arquivos Brasileiros de Cardiologia, vol. 92, no. 1, pp. 54-62, 2009.

[35] T. Inoue, Y. Sakai, S. Morooka et al., "Coronary flow reserve in patients with dilated cardiomyopathy," The American Heart Journal, vol. 125, no. 1, pp. 93-98, 1993.

[36] D. Neglia, O. Parodi, M. Gallopin et al., "Myocardial blood flow response to pacing tachycardia and to dipyridamole infusion in patients with dilated cardiomyopathy without overt heart failure: a quantitative assessment by positron emission tomography," Circulation, vol. 92, no. 4, pp. 796-804, 1995.

[37] O. Parodi, R. De Maria, L. Oltrona et al., "Myocardial blood flow distribution in patients with ischemic heart disease or dilated cardiomyopathy undergoing heart transplantation," Circulation, vol. 88, no. 2, pp. 509-522, 1993.

[38] E. I. Skalidis, F. I. Parthenakis, A. P. Patrianakos, M. I. Hamilos, and P. E. Vardas, "Regional coronary flow and contractile reserve in patients with idiopathic dilated cardiomyopathy," Journal of the American College of Cardiology, vol. 44, no. 10, pp. 2027-2032, 2004.

[39] R. Laguens, P. Alvarez, C. Vigliano et al., "Coronary microcirculation remodeling in patients with idiopathic dilated cardiomyopathy," Cardiology, vol. 119, no. 4, pp. 191-196, 2011.

[40] D. Abraham, R. Hofbauer, R. Schäfer et al., "Selective downregulation of VEGF-A ${ }_{165}$, VEGF- $\mathrm{R}_{1}$, and decreased capillary density in patients with dilative but not ischemic cardiomyopathy," Circulation Research, vol. 87, no. 8, pp. 644-647, 2000.
[41] L. Bolognese, N. Carrabba, G. Parodi et al., "Impact of microvascular dysfunction on left ventricular remodeling and long-term clinical outcome after primary coronary angioplasty for acute myocardial infarction," Circulation, vol. 109, no. 9, pp. 1121-1126, 2004.

[42] L. Galiuto, F. A. Gabrielli, A. Lombardo et al., "Reversible microvascular dysfunction coupled with persistent myocardial dysfunction: implications for post-infarct left ventricular remodelling," Heart, vol. 93, no. 5, pp. 565-571, 2007.

[43] T. Geshi, A. Nakano, H. Uzui et al., "Relationship between impaired microvascular function in the non-infarct-related area and left-ventricular remodeling in patients with myocardial infarction," International Journal of Cardiology, vol. 126, no. 3, pp. 366-373, 2008.

[44] X. Y. Hu, W. X. Wang, M. J. Yu, and et al, “Tongxinluo promotes mesenchymal stem cell tube formation in vitro," Journal of Zhejiang University: Science B, vol. 12, no. 8, pp. 644-651, 2011. 


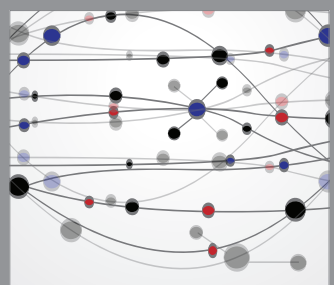

The Scientific World Journal
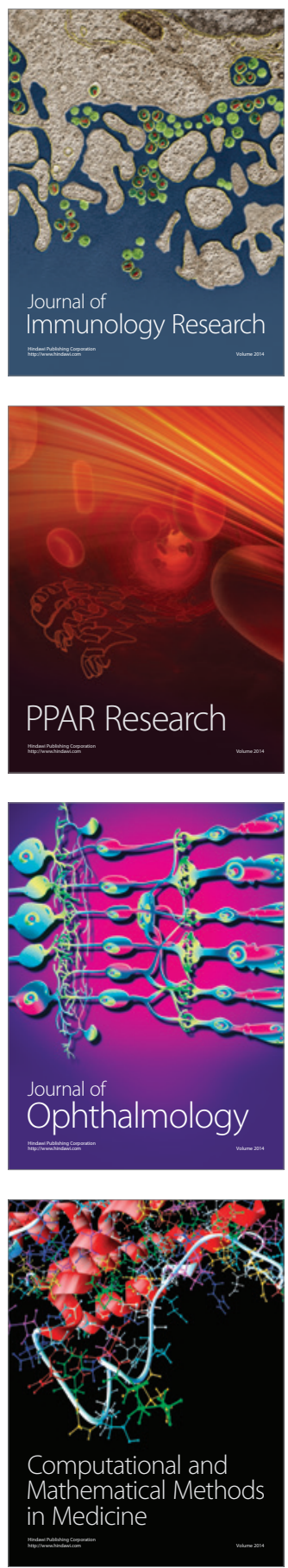

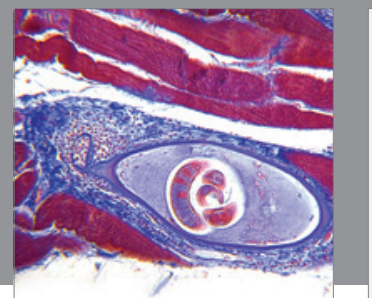

Gastroenterology

Research and Practice
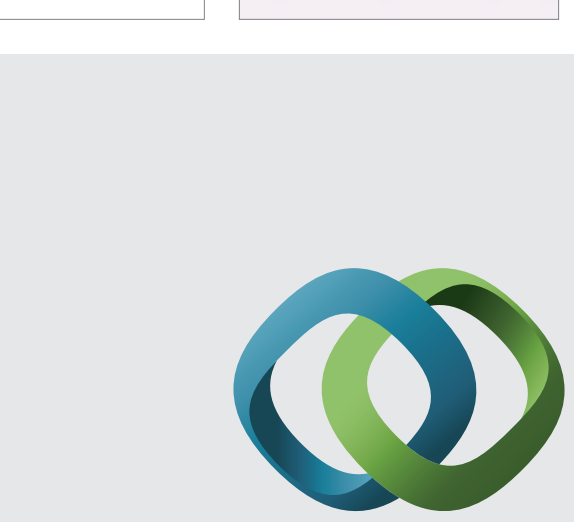

\section{Hindawi}

Submit your manuscripts at

http://www.hindawi.com
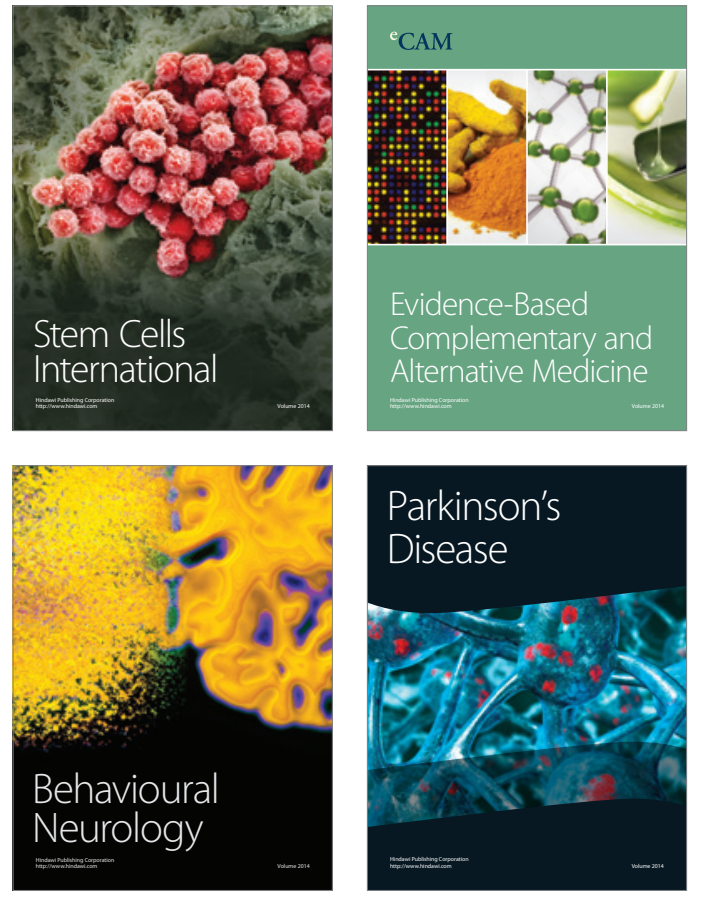
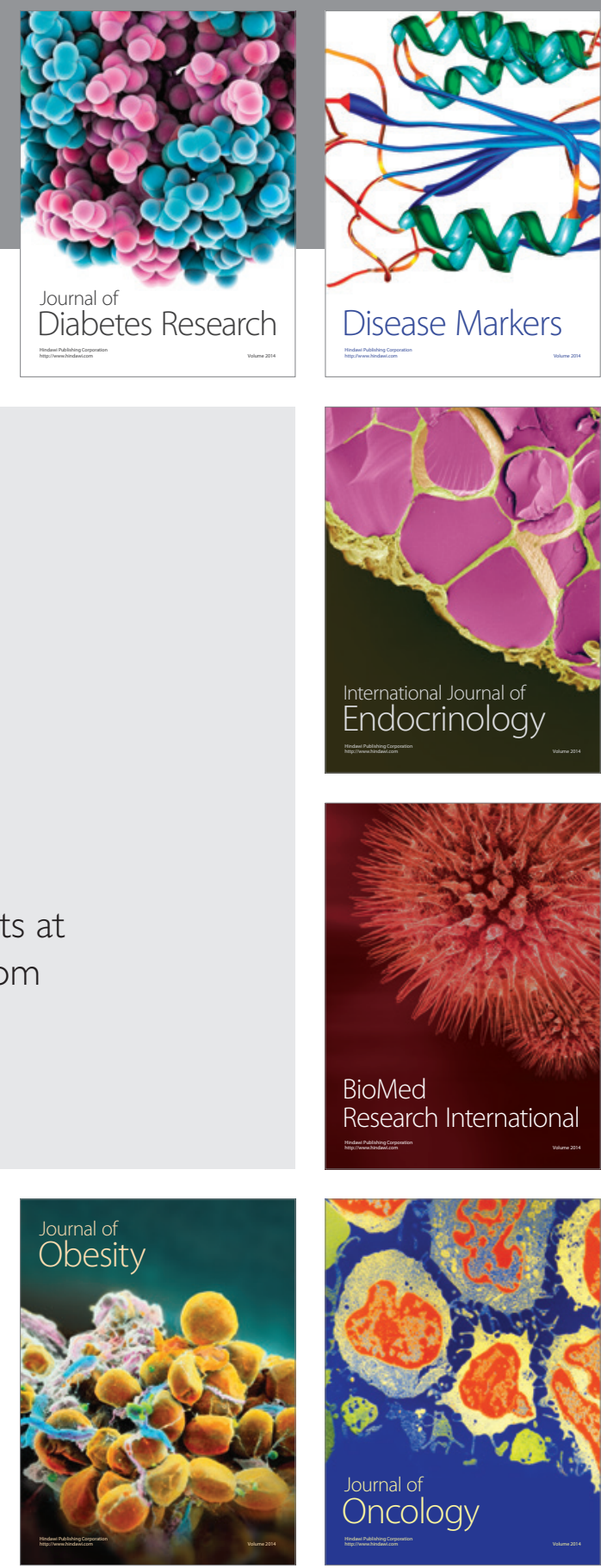

Disease Markers
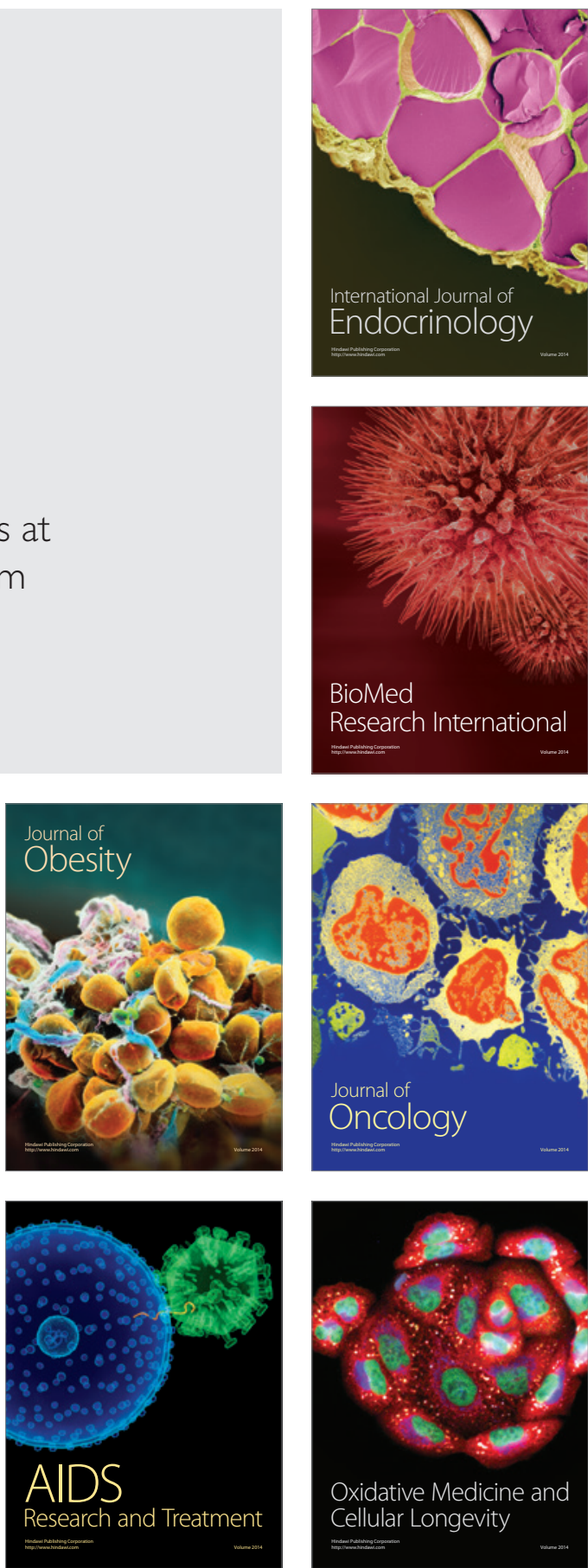